\title{
On Kinetically vs. Energetically Driven Growth Instabilities in Solid and Vapor Phase Epitaxy
}

\section{Citation}

Aziz, Michael J. 2000. On kinetically vs. energetically driven growth instabilities in solid and vapor phase epitaxy. Materials Research Society Symposium Proceedings 618.

\section{Published Version}

http://www.mrs.org/s_mrs/sec_subscribe.asp?

$D I D=218322 \& C I D=17120 \& S I D=1 \& V I D=113 \& R T I D=0$

\section{Permanent link}

http://nrs.harvard.edu/urn-3:HUL.InstRepos:2797692

\section{Terms of Use}

This article was downloaded from Harvard University's DASH repository, and is made available under the terms and conditions applicable to Other Posted Material, as set forth at http:// nrs.harvard.edu/urn-3:HUL.InstRepos:dash.current.terms-of-use\#LAA

\section{Share Your Story}

The Harvard community has made this article openly available.

Please share how this access benefits you. Submit a story.

Accessibility 


\title{
ON KINETICALLY VS. ENERGETICALLY DRIVEN GROWTH INSTABILITIES IN SOLID AND VAPOR PHASE EPITAXY
}

\author{
MICHAEL J. AZIZ \\ Division of Engineering and Applied Sciences, Harvard University, Cambridge MA 02138
}

\begin{abstract}
A stress-induced kinetically-driven morphological instability is of general applicability to driven systems. The effect of stress on the reaction mobility for incorporation into the growing solid couples to stress variations along a perturbed planar growth front, resulting in amplification or decay of the perturbation depending on the sign of the stress. Experimentally we studied a model system in which stress is applied externally to a chemically pure substance, permitting us to isolate the effect of strain from any possible effects of composition, and found that the new kinetically-driven effect dominates the behavior for solid phase epitaxial growth (SPEG) of $\mathrm{Si}(001)$. A linear stability analysis of a sinusoidally perturbed planar growth front, incorporating both the kinetically and the energetically driven effects, has been performed. Stability maps are developed, indicating parameter ranges under which the morphological evolution is dominated by the energetically-driven instability, the kinetically-driven instability, and the kineticallydriven stabilization. Numerical values of the key dimensionless parameters for SPEG and for $\mathrm{SiGe} / \mathrm{Si}(001)$ Molecular Beam Epitaxy (MBE) are very similar in cases where they are known, indicating that the kinetically-driven effect may be important in determining morphological evolution related to quantum dot formation in MBE as well.
\end{abstract}

\section{INTRODUCTION}

The morphological stability of surfaces and interfaces in elastically stressed solids is of great current interest. Much recent activity has focused on the morphological evolution of strained thin films coherently lattice matched to a bulk substrate. Under some circumstances, such films form an array of "quantum dots" which are of interest for their potential unique electronic properties. Control of the size and distribution of dots will be necessary for these properties to be fully exploited, but the interplay of the various physical and chemical effects determining the morphology is still controversial.

The focus of most work has been to understand and account for stress effects on the energetics, or driving forces, for these processes, one result of which is an elastic strain energy driven morphological instability on the surface of strained solids [1-4]. A uniaxially strained elastic solid in contact with a fluid across a flat interface has a spatially uniform stress and elastic strain energy density. A shape perturbation in the interface (Fig. 1) results in stress concentrations at the troughs and stress relaxation at the peaks and, because the chemical potential contains a term proportional to strain energy density, the chemical potential at the peak is reduced and that at the trough in increased. Atoms tend to spontaneously move from the troughs to the peaks, thereby amplifying the perturbation. Opposing this process is the effect of capillarity (assumed isotropic), but for perturbation wavenumbers below a critical value the elastic strain energy decrease is sufficient to "pay" for the increased surface energy, and the perturbation spontaneously grows. Some details depend on the mass transport pathway. The kinetics have been examined for transport by bulk diffusion [1, 5-7], surface diffusion [3, 8, 9], and evaporation-condensation [3, 10-12]. The mass transport pathway does not alter the critical wavenumber of the instability; it alters only the dependence of the amplification rate on wavenumber. 
All of this work assumes that the relevant transport coefficient (the surface diffusion coefficient for surface diffusion limited growth, or the reaction mobility or step-edge attachment rate constant for interface reaction limited growth) is independent of stress. We have previously called attention to the mobilities of the interfaces or atoms involved in growth $[13,14]$ and characterized the stress-dependence of mobilities [14-18], and we and others have predicted how the changes in mobility due to stress also help determine the growth morphology [5-7, 10-12, 16, $19,20]$.

Within transition state theory, the dependence upon stress $\sigma$ of an atomic or interfacial mobility $M$ is characterized by the activation strain tensor [14]

$$
V_{i j}^{*} \equiv k T \frac{\partial \ln M}{\partial \sigma_{i j}}
$$

A positive (negative) $V_{11}^{*}$, for example, implies that $M$ is reduced (enhanced) upon the application of a compressive $\sigma_{11}$. Just such a dependence of the interfacial mobility on stress has been observed $[14,16]$ in SPEG of $\operatorname{Si}(001)$, characterized by $V_{11}^{*}=+0.14$ times the atomic volume $\Omega$ of crystalline $\mathrm{Si}$.

A positive $V_{11}^{*}$ gives rise to a kinetically driven morphological instability during growth under compressive (i.e., negative) $\sigma_{11}$. For a sinusoidally perturbed interface between a growing crystalline phase and a fluid or amorphous parent phase in which stress is at least partially relaxed, or between two elastic phases in which the growing phase has higher moduli, there is a stress concentration in the trough and a stress relaxation at the peak of the perturbation, as shown in Fig. 1. The stress concentration in the trough results in a local interfacial mobility that is smaller than that at the peak; hence the peak grows faster and the perturbation tends to amplify. I call this the Barvosa-Carter ("BC") instability [10, 16].

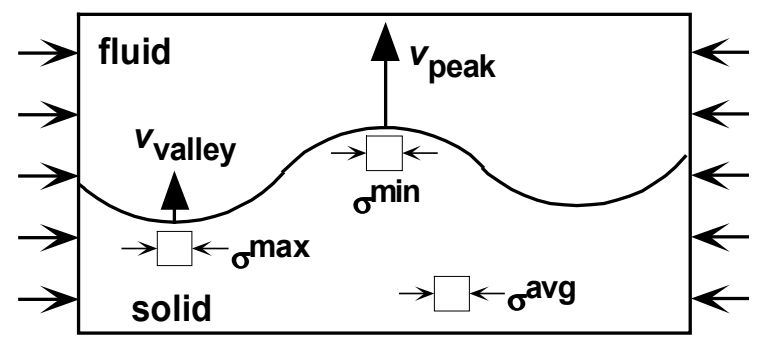

Fig. 1. Growth instabilities. Compressive stress is relieved in fluid and at peaks in solid, and concentrated in solid at troughs. Asaro-Tiller energetically-driven instability: peaks grow faster because elastic strain energy relief makes chemical potential at peaks lower than at troughs. BarvosaCarter kinetically-driven instability: stress concentration in troughs raises barrier to incorporation into growing phase more than does reduced stress at peaks; peaks run away. If stress reduces barrier instead, then trough catches up, yielding stability.

The stress effect on mobility is first order in stress, thus giving rise to opposite amplification behavior under tension and compression. In contrast, the elastic strain energy in the energetically-driven Asaro-Tiller ("AT") instability [1] is second order in stress, and therefore is destabilizing under both tension and compression. Hence if the interface is unstable under compression, which is generally observed in many growth situations and specifically known for solid-phase epitaxial growth (SPEG) of $\mathrm{Si}(001)[10,16]$ from an amorphous parent phase, and the stress-dependence of the mobility is the predominant factor in determining interface instability, then the interface should be stable in tension. This is indeed what we see in the 
laboratory. Our experimental work $[10,20]$ has demonstrated that the morphological stability or instability in SPEG is dominated by the stress dependence of the mobility and not by the energetics of elastic strain energy reduction. We avoided the potential complications associated with the coupling of composition to bulk and surface stress by studying a single-component system in which stress is applied externally. SPEG is analogous to growth from the vapor by evaporation-condensation: the chemical potential in the fluid phase is spatially uniform, and the magnitude of the discontinuity at the interface varies from peak to trough.

\section{LINEAR STABILITY ANALYSES}

The organizers of this symposium invited this paper in order to engender a discussion of whether the $\mathrm{BC}$ instability may also be important for quantum dot formation in growth from the vapor. In that spirit, please forgive me if I over-generalize and speculate about behavior in regimes beyond the range of validity of the analyses that have been performed to date. The linear stability of a sinusoidally perturbed interface growing with elastic strain contributions to both the energetics and a mobility has been analyzed only recently [5-7, 11, 12]. In general, these analyses are for stressed bulk solids rather than strained films on unstrained substrates and they neglect any anisotropy in the mobility, interfacial tension, and elastic constants (however, $\mathrm{Yu}$ and Suo [11] included elastic anisotropy in 2+1 dimensions). Here I focus on identifying and evaluating dimensionless groups arising from the dispersion relation derived by Voorhees and Aziz [12] for interface reaction-limited growth or evaporation-condensation kinetics. Although a case involving surface diffusion, which would be more relevant for Molecular Beam Epitaxy (MBE), has not been worked out, perhaps changing the transport pathway will not make vast qualitative differences in the results.

The dispersion relation of Voorhees and Aziz is

$$
\frac{r}{\bar{M}}=2 q Y(1-\nu)\left[-\tilde{G} \tilde{V} \tilde{\varepsilon}+\varepsilon^{2}+\frac{3 \varepsilon^{3} \tilde{V}}{\tilde{T}}\right]-q^{2} \Omega^{1 / 3} Y \tilde{\gamma}\left[1+\frac{2 \varepsilon \tilde{V}}{\tilde{T}}\right]
$$

where $r$ is the amplification rate of a perturbation of wavenumber $q ; \bar{M}$ is the interface mobility in the absence of stress; $Y$ and $v$ are the biaxial modulus and Poisson's ratio, respectively, of the growing solid; $\gamma$ is the surface or interfacial tension; $\varepsilon$ is the elastic strain and $\sigma_{a}=Y \varepsilon$ is the externally applied biaxial stress (positive for tensile in both cases). The behavior of the dispersion relation is determined by five dimensionless groups:

$$
\tilde{V} \equiv \frac{V_{11}^{*}}{\Omega} ; \tilde{G} \equiv \frac{-\Delta \mu_{\text {incorp }}}{k T} ; \varepsilon \equiv \frac{\sigma_{a}}{Y} ; \tilde{T} \equiv \frac{k T}{Y \Omega} ; \tilde{\gamma} \equiv \frac{\gamma}{\Omega^{1 / 3} Y}
$$

where $\Delta \mu_{\text {incorp }}$ is the chemical potential change per atom during the interface reaction and $k T$ has the usual meaning. In the three-term bracketed factor in Eq. (1), the first term is the BC term; the second is the raw AT term; and the second and third together describe the AT effect with a mobility modified by stress.

In Table I the dimensionless groups are evaluated for typical conditions of Si(001) SPEG and $\mathrm{Si}_{80} \mathrm{Ge}_{20} / \mathrm{Si}(001) \mathrm{MBE}$. It is seen that most of the dimensionless groups for the two processes are rather similar. However, we do not know the sign or magnitude of the activation strain tensor for $\mathrm{MBE}$, and the reported driving free energy is an upper limit. This is because the relevant chemical potential drop is not that from the 3D vapor to the bulk solid. Rather, it is only that portion of the chemical potential drop that occurs upon attachment at the step edge, as shown in 
Fig. 2. Because the stress-dependence of the terrace diffusion coefficient does not affect the stability of small perturbations [12], the chemical potential drop upon diffusing down any concentration gradient along a terrace to a step does not matter. Likewise, any chemical potential drop upon condensing from a 3D vapor to a $2 \mathrm{D}$ adatom or addimer vapor does not affect the stability. Because steps on $\mathrm{Si}(001)$ are highly "permeable", i.e. the mobile species are much more likely to traverse the step than to attach at the step (at least at $950{ }^{\circ} \mathrm{C}$ ) [21], the attachment barrier appears to be considerable and the chemical potential drop upon attachment is therefore also likely to be considerable. In Table I, we report an upper limit of 4.6 for the dimensionless driving force $(\tilde{G})$ in MBE, which was evaluated using $\Delta \mu_{\text {bulk }}=-k T \ln \left(p / p_{e}(T)\right)$, where $p$ is the equivalent 3D vapor pressure of the molecular beam and $p_{e}(T)$ is the pressure of the vapor that would be in equilibrium with the solid at the substrate temperature during growth. (For III-V MBE it seems that $\Delta \mu_{\text {bulk }}$ can be up to five times larger in magnitude [22]). Corresponding to the importance of the chemical potential drop on step-edge attachment, the dimensionless activation volume $(\tilde{V})$ for the MBE process represents the stressderivative of only the activation barrier for step-edge attachment and not that of the barrier for terrace diffusion.

Table I. Dimensionless groups evaluated for Si(001) SPEG and $\mathrm{Si}_{80} \mathrm{Ge}_{20} / \mathrm{Si}(001) \mathrm{MBE}$.

\begin{tabular}{|l|c|c|c|c|c|}
\hline & $\tilde{V} \equiv \frac{V_{11}^{*}}{\Omega}$ & $\tilde{G} \equiv \frac{-\Delta \mu_{\text {incorp }}}{k T}$ & $\varepsilon \equiv \frac{\sigma_{a}}{Y}$ & $\tilde{T} \equiv \frac{k T}{Y \Omega}$ & $\tilde{\gamma} \equiv \frac{\gamma}{\Omega^{1 / 3} Y}$ \\
\hline SPEG (Si) $[10,20]$ & +0.15 & 1.2 & \pm 0.0038 & 0.0041 & 0.011 \\
\hline $\mathrm{Si}_{80} \mathrm{Ge}_{20} \mathrm{MBE}[23]$ & $?$ & $\leq 4.6$ & -0.0088 & 0.0040 & 0.021 \\
\hline
\end{tabular}

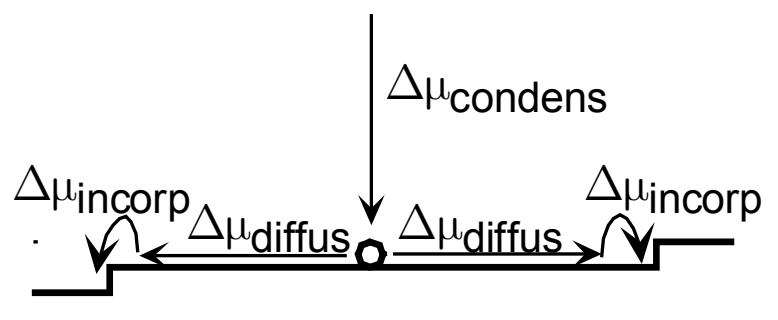

Fig. 2. Overall chemical potential drop $\Delta \mu_{\text {bulk }}$ from bulk vapor to bulk solid is sum of several contributions: $\Delta \mu_{\text {condens }}$ from $3 D$ vapor to $2 D$ vapor; $\Delta \mu_{\text {diffus }}$ from mid-terrace to step edge; $\Delta \mu_{\text {incorp }}$ upon incorporation into solid. Only $\Delta \mu_{\text {incorp }}$ enters into $\tilde{G}$. Any differences between so-called "type $A$ " and "type B" steps, as well as asymmetries in $\Delta \mu_{\text {incorp }}$ at up-steps and down-steps, are beyond the scope of this discussion.

\section{DISCUSSION}

Let us now consider the combinations of dimensionless parameters under which the AT instability dominates, the $\mathrm{BC}$ instability dominates, and the $\mathrm{BC}$ effect stabilizes a flat interface even in the presence of the destabilizing influence of the AT effect. It turns out that for this purpose the numerical value of $\tilde{\gamma}$ is irrelevant. This parameter affects only the highwavenumber behavior and the tradeoffs we are about to discuss depend solely on the lowwavenumber behavior. 
The $\mathrm{BC}$ term is a stabilizing influence when $\tilde{V} \varepsilon>0$, e.g. a positive $V_{11}^{*}$ (as in SPEG) and tensile stress. This stabilizing influence overwhelms the AT destabilization for all wavenumbers ("absolute stability") when

$$
\tilde{G}>\frac{\varepsilon}{\tilde{V}}+3 \frac{\varepsilon^{2}}{\tilde{T}} .
$$

From Eq. (2), a map of the regions of $(\varepsilon, \tilde{G})$-space dominated by the AT-driven instability and the BC-driven absolute stability effects is presented in Fig. 3. It is seen that for a wide range of $\tilde{V}$, the BC-driven stability should dominate the morphological evolution provided $\tilde{V} \varepsilon>0$.

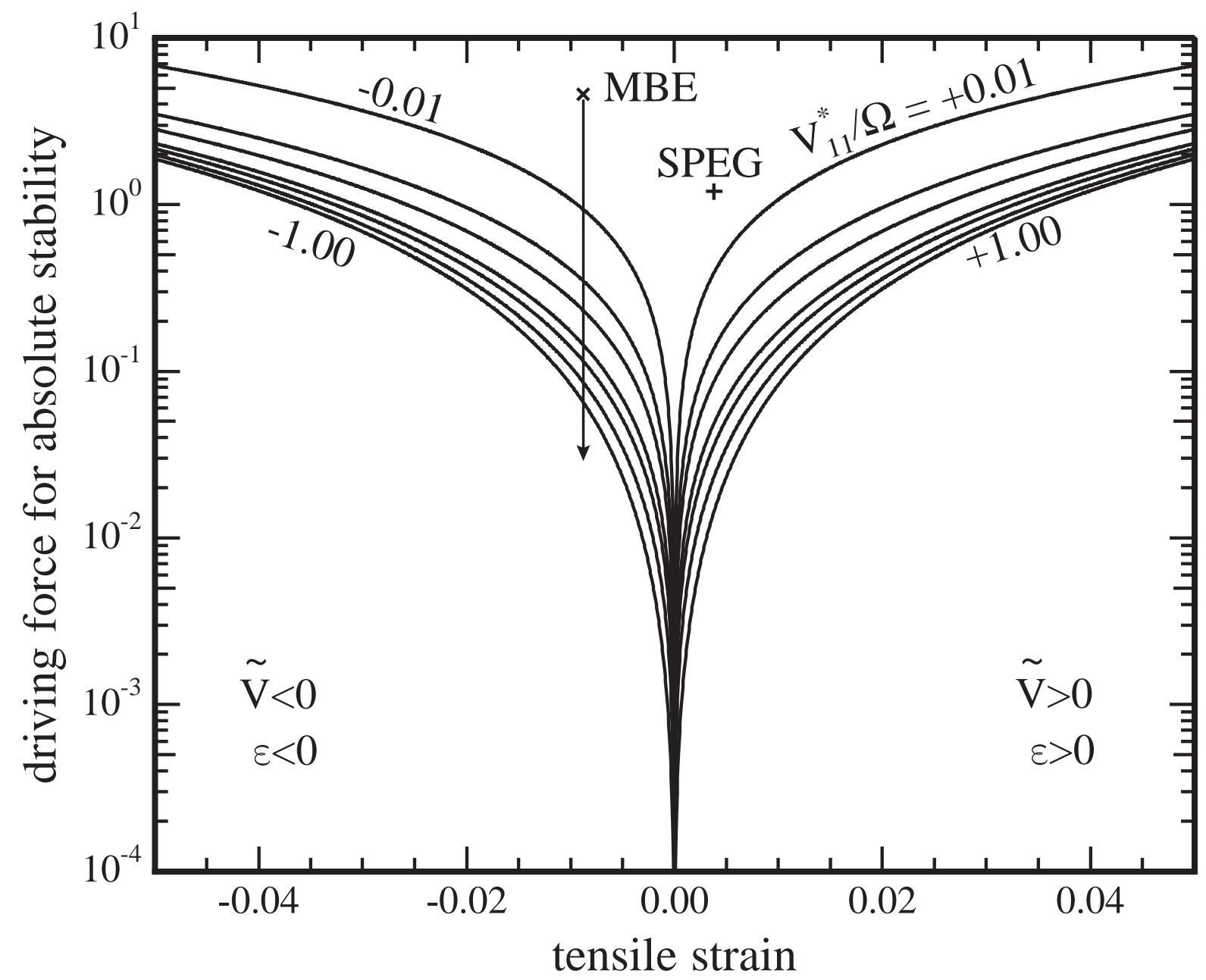

Fig. 3. Critical value of $\tilde{G}$ required for absolute stability when $\tilde{V} \varepsilon>0$, from Eq. (2). $\tilde{T}=0.0041$. Below each line the AT instability dominates; above each line the BC effect causes absolute stability. Lines are parameterized by $|\tilde{V}|=0.01,0.03,0.05,0.10,0.15,0.30$, and 1.00

from top to bottom; on the right-hand side $\tilde{V}>0$ and on the left-hand side $\tilde{V}<0$. Cross indicates SPEG experimental conditions. " $x$ " indicates upper limiting value for $\mathrm{Si}_{80} G e_{20} \mathrm{MBE}$ experiments discussed in text. 
The $\mathrm{BC}$ term is a destabilizing influence when $\tilde{V} \varepsilon<0$, e.g. a positive $V_{11}^{*}$ (as in SPEG) and compressive stress. The $\mathrm{BC}$ effect dominates the instability when

$$
\tilde{G}>-\frac{\varepsilon}{\tilde{V}}-3 \frac{\varepsilon^{2}}{\tilde{T}}
$$

From Eq. (3), a map of the regions of $(\varepsilon, \tilde{G})$-space dominated by the AT-driven instability and the BC-driven instability effects is presented in Fig. 4. The boundary curve identified with $\tilde{V}=+0.15$ is the relevant one for SPEG; one sees that the actual driving free energy for SPEG places the process well into the BC-dominated regime. Note that if the actual value of $\tilde{V}$ for $\mathrm{Si}_{80} \mathrm{Ge}_{20} \mathrm{MBE}$ were even as low as +0.05 , the transition from AT-dominated to BC-dominated instability occurs at $\tilde{G} \approx 0.1$, which is less than $1 / 4$ of the bulk (3D vapor $\rightarrow 3 \mathrm{D}$ solid) driving force. From this model it seems that unless both the first diagonal component of the activation strain tensor and the chemical potential drop upon step-edge attachment are quite small, the BC effect could indeed be important in MBE.

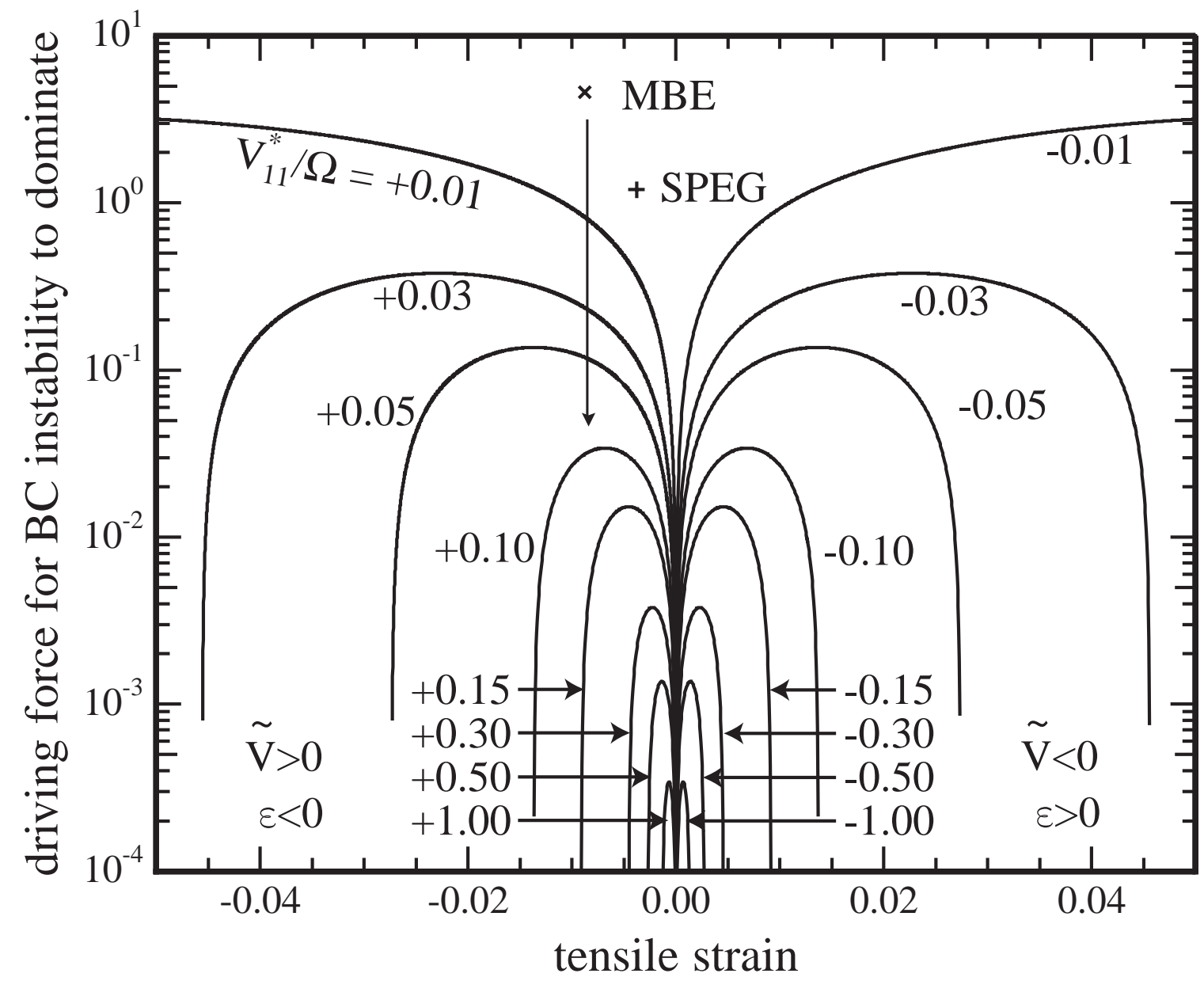

Fig. 4. Critical value of $\tilde{G}$ required for $B C$-dominated instability when $\tilde{V} \varepsilon<0$, from Eq.(3). $\tilde{T}=0.0041$. Below each line, parameterized by $\tilde{V}$, the AT instability dominates; above each line the BC instability dominates. Cross indicates SPEG experimental conditions. " $x$ " indicates upper limiting value for $\mathrm{Si}_{80} \mathrm{Ge}_{20} \mathrm{MBE}$ experiments discussed in text. 


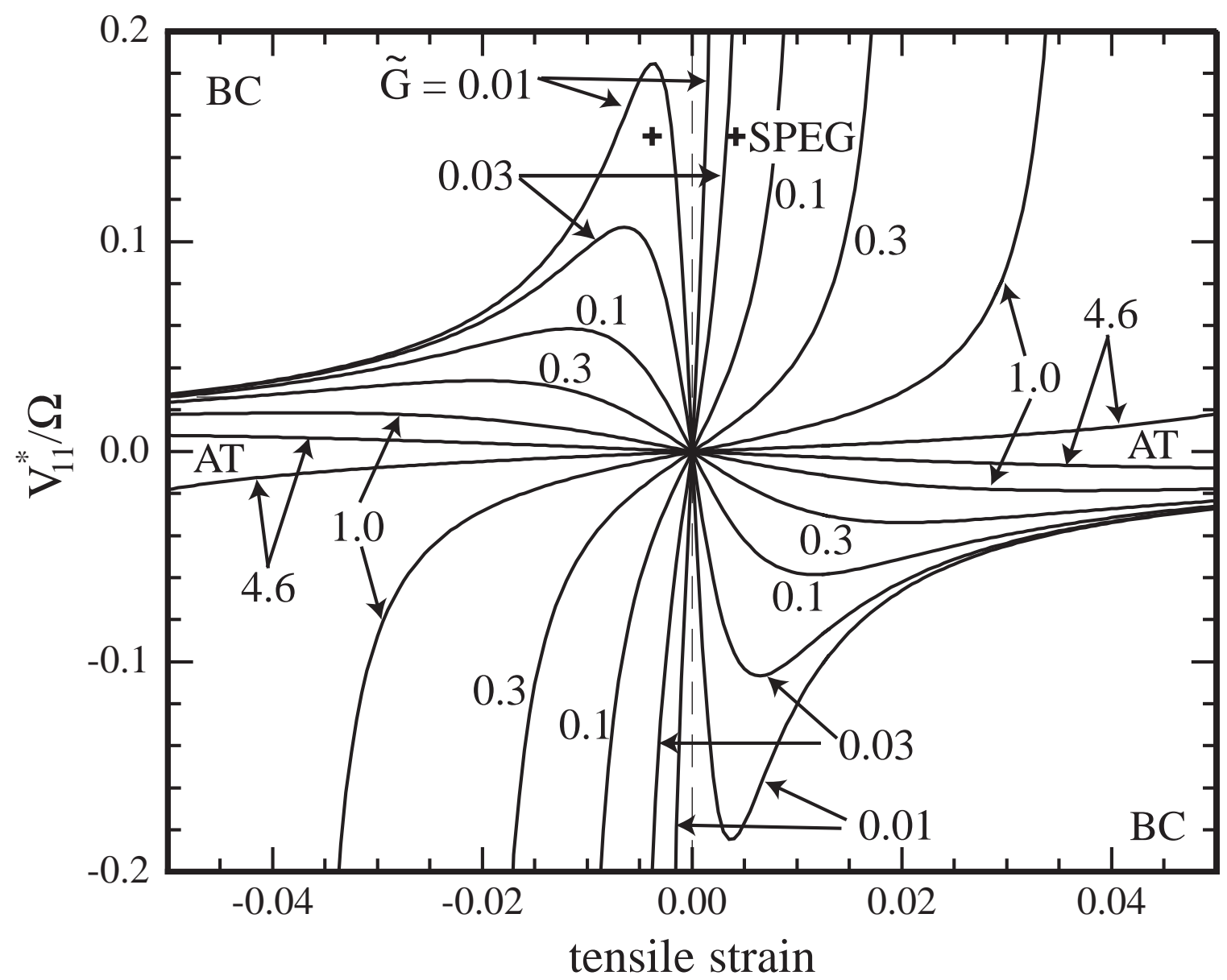

Fig. 5. Critical value of $\tilde{V}$ required for BC-dominated stability (1st and $3 r d$ quadrants) and BCdominated instability (2nd and 4th quadrants), from Eqs. (4) and (5). The AT instability dominates between each line, parameterized by $\tilde{G}$, and the horizontal axis. Outside the lines we find BC-induced absolute stability (1st and 3rd quadrants) or BC-dominated instability (2nd and 4th quadrants). $\tilde{T}=0.0041$. Crosses indicate location of SPEG experiments.

The various regimes can all be combined into a stability map in $(\varepsilon, \tilde{V})$-space as shown in Fig. 5. The curves in this figure identify how large in magnitude $\tilde{V}$ has to be, for various values of $\tilde{G}$, for the AT instability to give way to the BC-dominated stability (1st and 3rd quadrants) or instability (2nd and 4th quadrants). In the 1 st and 3rd quadrants, BC-dominated absolute stability occurs when

$$
|\tilde{V}|>\left|\frac{\varepsilon}{\tilde{G}-3 \frac{\varepsilon^{2}}{\tilde{T}}}\right| .
$$

In the 2nd and 4th quadrants, $\mathrm{BC}$-dominated instability occurs when 


$$
|\tilde{V}|>\left|\frac{\varepsilon}{\tilde{G}+3 \frac{\varepsilon^{2}}{\tilde{T}}}\right| .
$$

When $\tilde{V}=0$ or $\tilde{G}=0$ we have only the AT instability in both tension and compression, as expected. But as $\tilde{G}$ increases, the "wedges" of AT-dominated behavior (identified with "AT" in Fig. 5) become restricted to smaller and smaller values of $|\tilde{V}|$.

\section{SUMMARY}

A kinetically-driven growth instability in stressed, driven systems is favored over the energetically-driven instability for large values of the chemical potential drop upon incorporation into the solid and nonnegligible magnitudes of the relevant component of the activation strain tensor. The kinetically-driven term is destabilizing when $\tilde{V} \varepsilon<0$ and stabilizing when $\tilde{V} \varepsilon>0$. We have used SPEG of pure $\mathrm{Si}(001)$, strained by the application of external loading, as a "clean" system for investigating growth instabilities and have demonstrated that the kinetically-driven effect causes stability in tension and dominates the observed instability in compression. The simplistic calculations presented here for alloy growth neglect many complicating factors such as the interactions of composition and stress [24]; the possible asymmetries due to differential atomic mobilities [25] and preferential segregation of one alloy species [26]; and anisotropy of surface tension, surface diffusivity, reaction barrier, and elastic strain energy. However, from the results of these calculations it appears that the kinetically-driven effect could be important for other growth processes besides SPEG, such as semiconductor alloy heteroepitaxy and the formation of quantum dots. For further insight, the dispersion relation for growth in the presence of surface diffusion and step-incorporation barriers will have to be developed, and the parameters $\tilde{V}$ and $\tilde{G}$ will have to be determined.

\section{ACKNOWLEDGMENTS}

I thank Peter Voorhees for his efforts as a collaborator on this topic and Jerry Floro for communicating the relevant growth parameters for $\mathrm{Si}_{80} \mathrm{Ge}_{20} \mathrm{MBE}$. This research was funded by NSF-DMR-98-13803.

\section{REFERENCES}

1 R. J. Asaro and W. A. Tiller, Metall. Trans. 3, 1789 (1972).

2 M. A. Grinfeld, Sov. Phys. Dokl. 31, 831 (1986).

3 D. J. Srolovitz, Acta Metall. 37, 621 (1989).

4 V. A. Shchukin and D. Bimberg, Rev. Mod. Phys. 71, 1125 (1999).

5 G. Grinstein, Y. Tu, and J. Tersoff, Phys. Rev. Lett. 81, 2490 (1998).

6 V. P. Zhdanov, Phys. Rev. Lett. 83, 656 (1999).

7 G. Grinstein, Y. Tu, and J. Tersoff, Phys. Rev. Lett. 83, 657 (1999).

8 J. Gao, Int. J. Solids Structures 28, 703 (1991).

9 B. J. Spencer, P. W. Voorhees, and S. H. Davis, J. Appl. Phys. 73, 4955 (1993).

10 W. Barvosa-Carter, M. J. Aziz, L. J. Gray, and T. Kaplan, Phys. Rev. Lett. 81, 1445 (1998). 
11 H. H. Yu and Z. Suo, J. Appl. Phys. 87, 1211 (2000).

12 P. W. Voorhees and M. J. Aziz, in Conference on Interfaces for the Twenty-First Century, edited by M. K. Smith and G. B. McFadden (Imperial College Press, 2000).

13 M. J. Aziz, Appl. Phys. Lett. 70, 2810 (1997).

14 M. J. Aziz, P. C. Sabin, and G.-Q. Lu, Phys. Rev. B 44, 9812 (1991).

15 M. J. Aziz, S. Circone, and C. B. Agee, Nature 390, 596 (1997).

16 W. Barvosa-Carter and M. J. Aziz, Mater. Res. Soc. Symp. Proc. 441, 75 (1997).

17 N. Bernstein, M. J. Aziz, and E. Kaxiras, Phys. Rev. B 58, 4579 (1998).

18 W. B. Carter and M. J. Aziz, Mat. Res. Soc. Symp. Proc. 356, 87 (1995).

19 J. Tersoff, Y. Tu, and G. Grinstein, Appl. Phys. Lett. 73, 2328 (1998).

20 J. F. Sage, W. Barvosa-Carter, and M. J. Aziz, Appl. Phys. Lett. 77, in press (2000).

21 S. Tanaka, N. C. Bartelt, C. C. Umbach, R.M. Tromp and J.M. Blakely, Phys. Rev. Lett. 78, 3342 (1997).

22 H. Seki and A. Koukitu, J. Cryst. Growth 78, 342 (1986).

23 J.A. Floro (personal communication).

24 J. E. Guyer and P. W. Voorhees, Phys. Rev. B 54, 11710 (1996).

25 B. J. Spencer, P. W. Voorhees, and J. Tersoff, Appl. Phys. Lett. 76, 3022 (2000).

26 C. Priester and G. Grenet, J. Vac. Sci. Technol. B 16, 2421 (1998). 